\title{
Topology Optimization of Automotive Sheet Metal Part using Altair Inspire
}

\author{
F. NETSANET \\ University of Debrecen, Faculty of Engineering, Department of Mechanical Engineering, \\ netsanetanteneh79@gmail.com /netsanet@mailbox.unideb.hu
}

\begin{abstract}
In an optimisation problem, different candidate solutions are compared with each other, and then the best or optimal solution is obtained which means that solution quality is fundamental. Topology optimisation is used at the concept stage of design. It deals with the optimal distribution of material within the structure.Altair Inspire software is the industry's most powerful and easy-to-use Generative Design/Topology Optimization and rapid simulation solution for design engineers. In this paper Topology optimization is applied using Altair inspire to optimize the Sheet metal Angle bracket. Different results are conducted the better and final results is fulfilling the goal of the paper which is minimizing the mass of the sheet metal part by 65.9\% part and Maximizing the stiffness with Better Results of Von-Miss Stress Analysis, Displacement and comparison with different load cases. This can lead to reduced costs, development time, material consumption, and product less weight.
\end{abstract}

Key words: Topology optimization , Factor of safty, Von miss stress, Displacement, Efficiency, mass

\section{Introduction}

Topology optimization is already widely used in order to get ideas for lightweight constructions. Sheet metals manufactured by deep drawing should have a constant wall thickness and no undercuts in punch direction. The topology optimisation deals with the optimal distribution of material within the structure. It is employed when the design is just conceived and is at the concept or preliminary stage. Unlike traditional optimisation, topological optimisation does not require design variables (that is, independent variables to be optimised)[1]. Altair Inspire software is the industry's most powerful and easy-to-use Generative Design/Topology Optimization and rapid simulation solution for design engineers. It enhances the concept development process by enabling simulation-driven design to increase your product's efficiency, strength and manufacturability. This can lead to reduced costs, development time, material consumption, and product weight. Easily generate dynamic motion of complex mechanisms, automatically identifying contacts, joints, springs and dampers.

In engineering, the optimisation of an objective function is basically the maximisation or minimisation of a problem subjected to constraints[2]. Regression functions can be effective tools in the solution of engineering optimization problems[3]. The application of Support Vector Machine (SVM) can handle the large number of data in a special way and helps to find the optimal design parameters.[4]Sheet metal structures for instance are typically used to create automotive components that provide significant structural support under high loads, such as bumpers, seat frames and body in white parts. Particular 
focus is consequently placed in the optimisation of automotive components to produce designs that both perform well and are cost-effective[5]. Finite element analysis is widely used in Automotive parts such as rubber product design to choose the appropriate material models and determine the related material parameters So that a products can suffer from large deformation upon working conditions while behaving as a non-linearly elastic, isotropic and incompressible material. Using test data and ANSYS for curve fitting process, the material constants for Mooney-Rivlin and Yeoh model have been established.[6] An angle bracket is a type of fastener that can also be referred to as a shelf bracket, corner brace or L bracket. Typically made of steel and stainless steel they are used to join two parts at a 90 degree angle. The brackets are suitable for bracing and supporting shelves and drawers and many other applications. Braced brackets are typically stronger than standard L-shaped brackets due to their additional support strut. Brackets lengths vary in both directions to accommodate each application as well as having a variety of widths and hole patterns.

\section{Materials and Methods}

Topological optimisation capabilities of a commercially available finite-element solver ANSYS 8.0 have been employed through APDL (ANSYS Parametric Design Language) to find the optimal layout of material in the brackets. Holes come in various shapes such as round and slotted and hole patterns vary depending on the bracket size and shape. Some brackets will feature countersunk holes for a clean and smooth finish. Computational topology optimisation is growing to be an increasingly reliable and necessary tool to improve quality and efficiency for development of a vast range of engineering components, for example in the manufacturing of sheet metal components in the automotive industry. A software package is developed which can design an appropriate topology of body structure of stamping die components with a reduced weight. This is done by implementing the evolutionary structural optimization algorithm. The final results show a reduction of $37 \%$ of volume and $8 \%$ of maximum displacement, respectively[7]. Generate optimized lattice and mixed solid - lattice structures, visualize simulation results in 3D, and export lattice designs in a .stl file format for 3D printing. View and interactively assign loads to load cases and import / export design loads in .csv file format with the new Load Cases Table.

A recipe of the operation for a final optimal design is then presented to the manufacturing engineers thus saving them time and cost optimal design is then presented to the manufacturing engineers thus saving them time and cost[2], [5], [8]. Design for additive manufacturing with overhang shape controls to help reduce overhangs to create more self-supporting structures Today, die design standards are used to design the structure of die components. These standards are usually based on high safety factors. So, the die components are often heavier and larger than required.

Research into design for additive manufacturing has mostly focused on the detailed design of (smaller) structural components, where the allowable design space and the loads are known and fixed. Most topology optimization methods are more suited for detailed design studies due to their cost and lowerresolution representations of the same optimization problem can be far off from the result. We therefore develop a design methodology specifically for early-stage design phases, with low computational cost and a good approximation of the final structural properties [9].[10], [11] All considered manufacturing constraints are heuristics, which are nondifferentiable, and govern the gradient based topology 
optimization approach. [12][16] Generate optimized lattice and mixed solid - lattice structures, visualize simulation results in 3D, and export lattice designs in a .stl file format for 3D printing. [17][19] View and interactively assign loads to load cases and import / export design loads in .csv file format with the new Load Cases Table. [20][24] Design for additive manufacturing with overhang shape controls to help reduce overhangs to create more self-supporting structure

\subsection{Modelling of Angle bracket}

Altair Inspire software is the industry's most powerful and easy-to-use Generative Design/Topology Optimization and rapid simulation solution for design engineers. It enhances the concept development process by enabling simulation-driven design to increase your product's efficiency, strength and manufacturability Altair Inspire software is the industry's most powerful and easy-to-use Generative Design/Topology Optimization and rapid simulation solution for design engineers The 3D CAD model of a selected angle bracket, this is original design part. Detail analysis and simulation is done in two different cases. The first one is when the load is outward and when the load is inward push and pull force the result is little bit different.
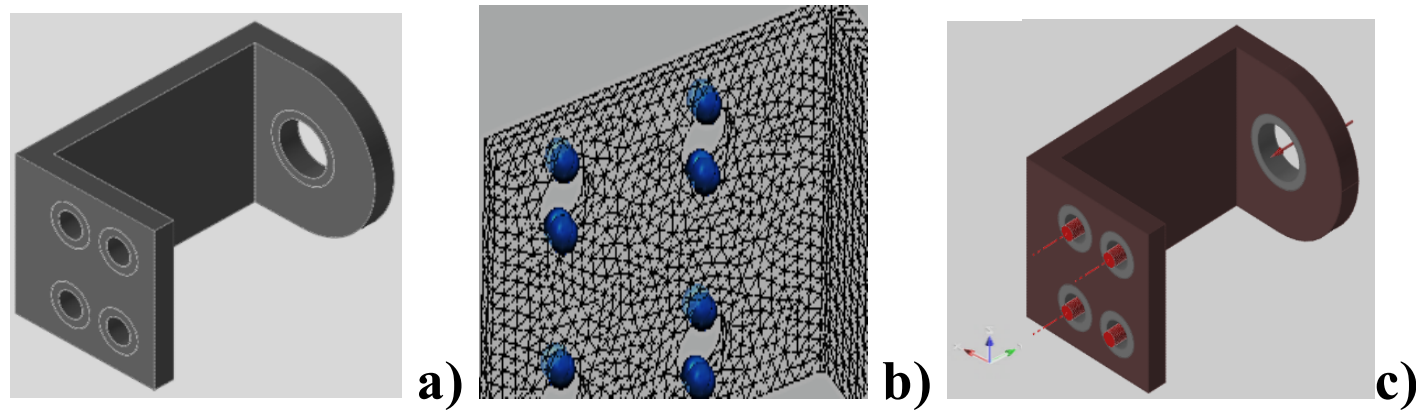

Figure 1. A),3D CAD Modelling B).mesh and C) Definend load and Support using Altair inspire

\section{Results and Discussion}

Applying the load to the $1 \mathrm{KN}$ on the sheet metal part and analysing the Displacement Vonmiss stress to checke whether it is ready enough to optimize and reduce the mass and increasing the efficiency as well,Finaly we have MAX and minimum value from the analysis max displacement $0.0368 \mathrm{~mm}$ and minimum displacement $2.656 \mathrm{e}-10 \mathrm{~mm}$.as we see the maximum deformation is prety small amount so we can optimise the material. When we see the Vonmiss stress we have max value $4.30 \mathrm{mpa}$ and min value $2.955 \mathrm{e}-04$ here we have to consider maximissing efficiency. Targeting to save the mass and keep the required stiffness, a topology optimization method is Applied. 

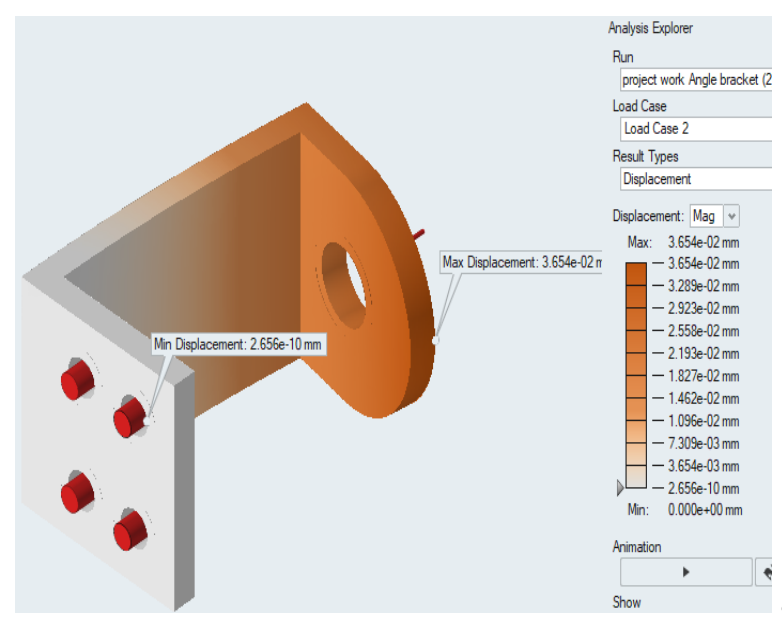

a)

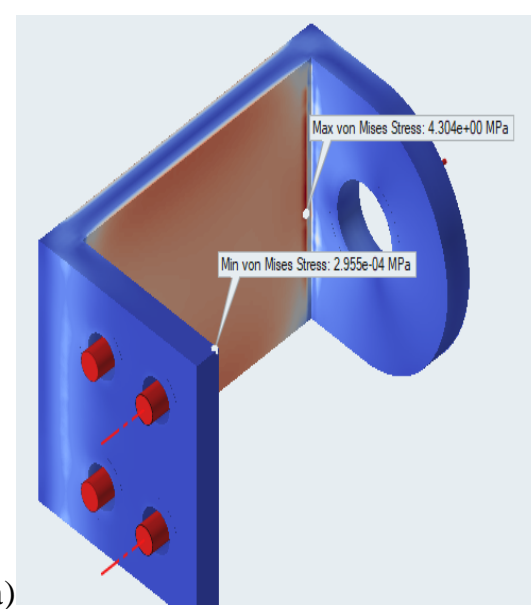

Figure 2). a) Displacement and b) Von-Miss stress analysis using Altair software before optimization

\subsection{Topology Optimization and Analysis}

The topology optimisation is used at the concept stage of design. In the concept stage, it is very difficult for the designer to search the design space to find the optimal material distribution or layout. This truss like structure can be optimised further for weight, considering the area of the truss members as design variable. Under various constraints like deformation, stresses in members. Comparing the direct optimization and Topology optimization there is agread difference between the amount of mass reduction and the efficiency as well.Here we can see the direct optimization result graph .

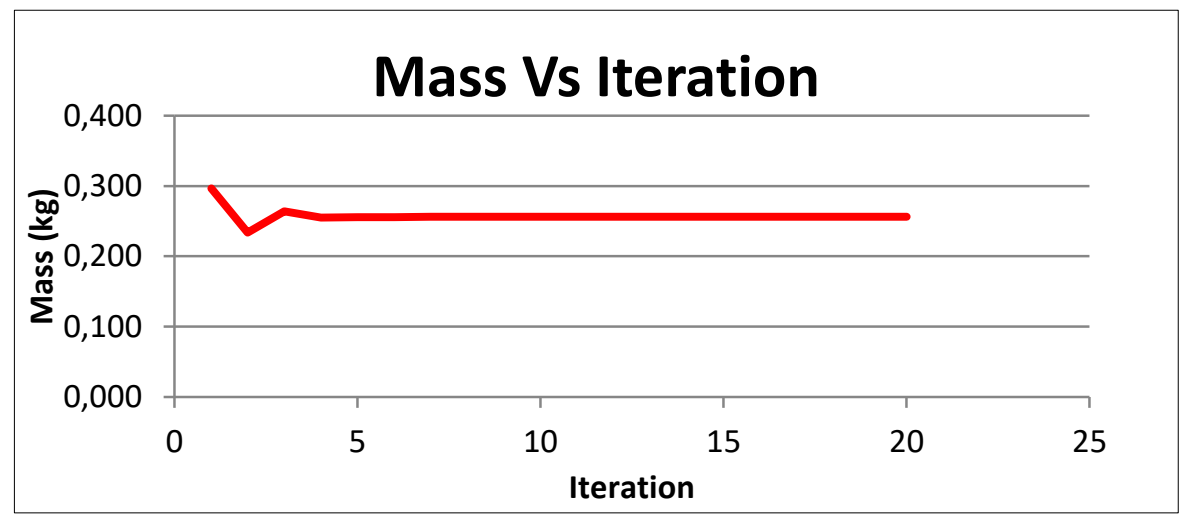

Figure 3. Simulation and optimization result Mass Vs number of iterations (total transition checked using Solid Edge software)

The above graph indicates How the mass is going to be lower and lower as we increase the iterations .Here we can see that the mass is minimised with approximately $60 \%$ for the iteration \#20 times, the result is different and we can take it the most precise with higher efficiency and lower mass .In this paper, Angle bracket is topologically optimised for loading conditions given $1 \mathrm{KN}$.The initial design space was a normal Angle bracket with masss of $0.75 \mathrm{~kg}$. The final shape converged to truss like structure. This approach is ideal for maximizing the stiffness of components while trying to achieve a desired mass target. It can also be used to minimize the mass of a model, depending on your optimization objective. If you maximize the stiffness of a design space, the resulting shape will resist deflection, but may be 
heavier as a consequence. If you minimize mass, the resulting shape will be light but may deflect more. When running a topology optimization, mass targets are used when maximizing stiffness, and stress constraints are used when minimizing mass.
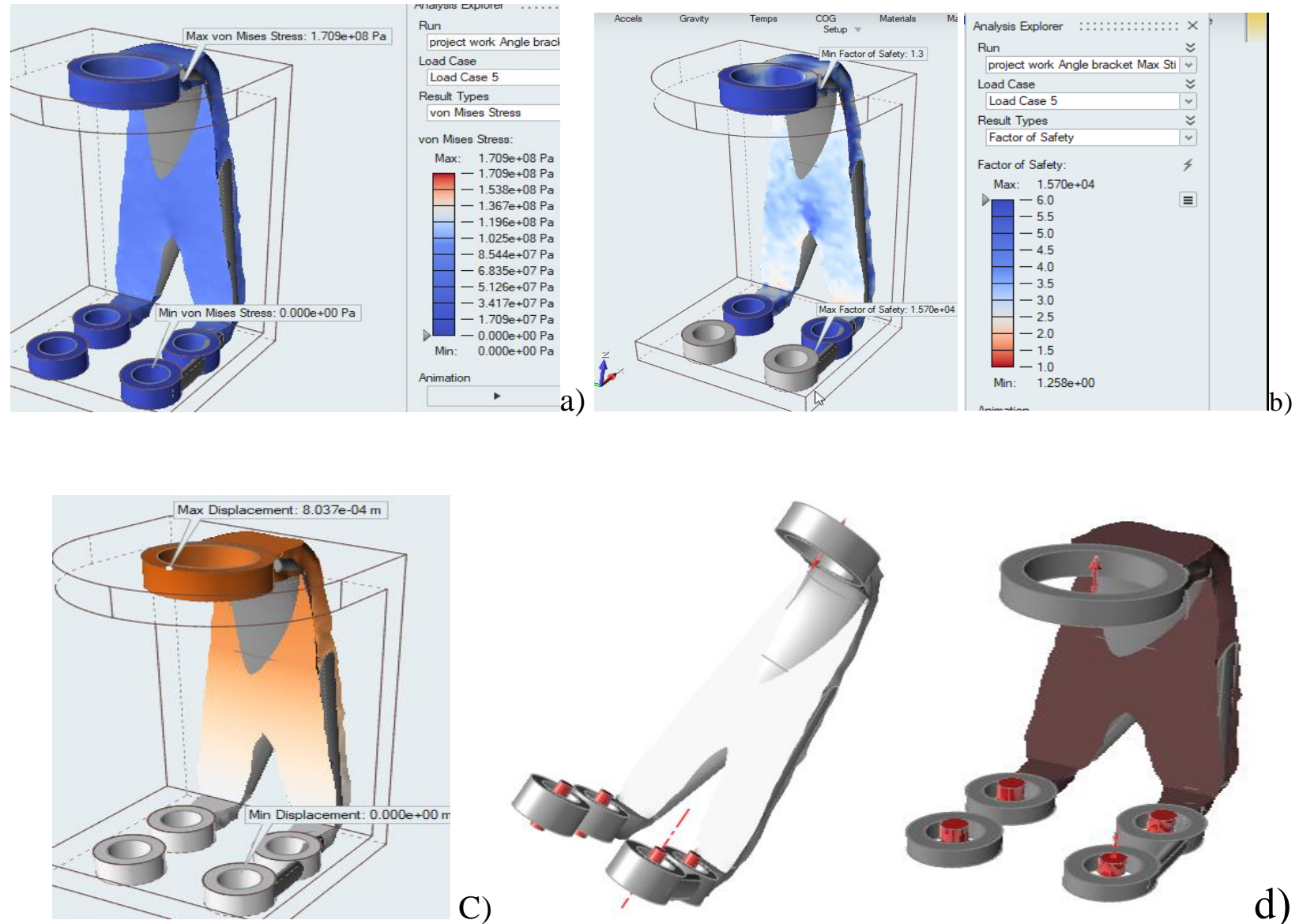

d)

Figure 4: Altair inspire Topology optimization and analysis of Angle bracket with Variable parameters;[a),Analysis of Von Miss stress Max and Min value; b) Factor of safety at MAX and minimum value; $\boldsymbol{c}$ )The total Displacement while applying the load and; $\boldsymbol{d}$ )the final optimized part with minimum mass and maximum Stiffness.]

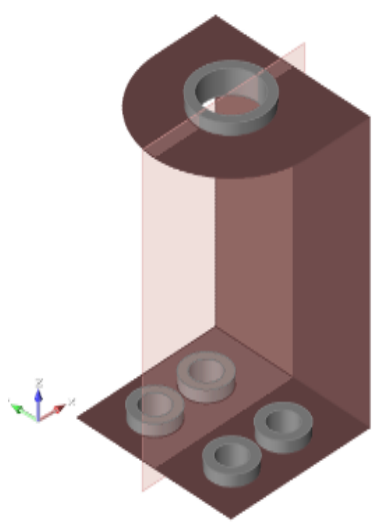

a)
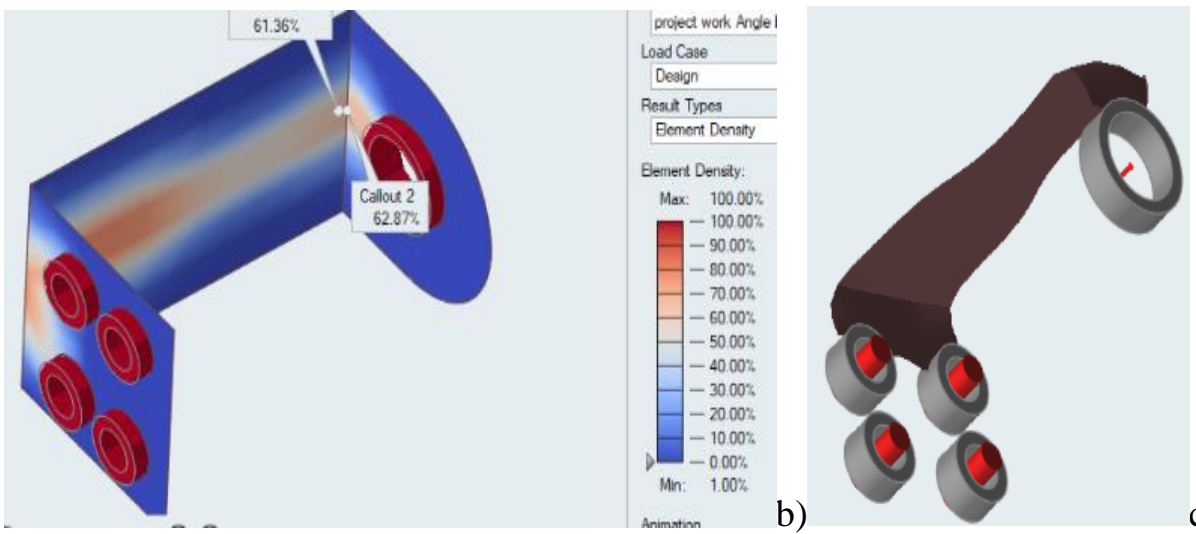

Figure 5 a) Altair inspire sheet metal mid surface part b) Analysis of Element density c) final sheet metal part after Applying Topology optimization on mid surface part. 


\section{Conclusion}

Introducing engineers to an entirely new software tool with a new user interface and potentially unfamiliar workflow could delay or hinder incorporation of topology optimization into industry design practice. So, to estimate the ease with which topology optimization software can be adopted, compatibility with non-native FEA platforms and the availability of online and on-site training was investigated. If time is to be invested in learning to use a new piece of software, then it should also be as multifunctional as possible to streamline the flow between analyses. Topology optimization is already widely used in order to get ideas for lightweight constructions. Altair Inspire software is the industry's most powerful and easy-to-use Generative Design/Topology Optimization and rapid simulation solution for design engineers Sheet metals manufactured is now adays has got optimised to decrees the amount of mass and maximise the stiffness.

Most topology optimization methods are more suited for detailed design studies due to their cost and lower-resolution representations of the same optimization problem can be far off from the result. Its proved that Develop a design methodology specifically for early-stage design phases, with low computational cost and a good approximation of the final structural properties. This paper also concludes in a specific part which is Angle bracket sheet metal part with initial mass of $0.76 \mathrm{~kg}$ and thickness $8 \mathrm{~mm}$. Having material properties of Steel with detail variables through Altair Software. The analysis has the value with $65.8 \%$ of mass reduction and high value of stiffness with best factor of safety of minimum 1.26. This can lead to reduced costs, development time, material consumption, and product less weight.

\section{References}

[1] Dienemann, R., Schumacher, A., Fiebig, S. (2016) 'Topology and shape optimization of sheet metals with integrated deep-drawing-simulation', Proc. of 12th World Conference on Computational Mechanics, Seoul, Korea.

[2] Gunwant, D., Misra, A. (2012) 'Topology Optimization of sheet metal brackets using ANSYS', MIT International Journal of Mechanical Engineering, 2(2), pp. 120-126.

[3] Kocsis, I., Mankovits, T. (2013) 'Application of non-parametric regression in engineering optimization', Analele Universitatii din Oradea Fasciola Management si Inginerie Tehnologica/Annals of the University of Oradea Fascicle of Management and Technological Engineering, 12(22), pp. 159-162.

[4] D. Huri and T. Mankovits (2019) 'Automotive rubber part design using machine learning', IOP Conf. Ser. Mater. Sci. Eng., vol. 659, p. 012022, doi: 10.1088/1757-899X/659/1/012022.

[5] M. Sehmi, J. Christensen, C. Bastien, and S. Kanarachos (2018) 'Review of topology optimisation refinement processes for sheet metal manufacturing in the automotive industry', Struct. Multidiscip. Optim., 58 (1), pp. 305-330. doi: 10.1007/s00158-017-1876-0.

[6] Huri D., Mankovits (2018) 'Comparison of the material models in rubber finite element analysis', IOP Conf. Ser. Mater. Sci. Eng., vol. 393, p. 012018. doi: 10.1088/1757-899X/393/1/012018. 
[7] Azamirad G, B. Arezoo (2017) 'Topology optimization of stamping die components using evolutionary structural optimization method', Proc. Inst. Mech. Eng. Part B J. Eng. Manuf., 231 (4) pp. 690-698. doi: 10.1177/0954405415597630.

[8] Berrocal L. et al. (2019) 'Topology optimization and additive manufacturing for aerospace components' Prog. Addit. Manuf., 4 (2) pp. 83-95. doi: 10.1007/s40964-018-0061-3.

[9] Opgenoord M. M. J., Willcox K. E (2019) 'Design for additive manufacturing: cellular structures in early-stage aerospace design', Struct. Multidiscip. Optim., 60 (2) pp. 411-428. doi: 10.1007/s00158-019-02305-8.

[10] Mankovits, T., Huri, D., Kállai, I., Kocsis, I., Szabó, T. (2014) ‘Material characterization and numerical simulation of a rubber bumper', International Journal of Mechanical, Aerospace, Industrial, Mechatronic and Manufacturing Engineering, 8(8), pp. 1367-1370.

[11][Mankovits, T., Budai, I., Balogh, G., Gábora, A., Kozma, I., Varga, T., . . Kocsis, I. (2014) 'Structural analysis and its statistical evaluation of a closed-cell metal foam', International Review of Applied Sciences and Engineering, 5(2), 135-143. doi:http://dx.doi.org/10.1556/IRASE.5.2014.2.5

[12]Patel, J., Campbell, M. I. (2010) 'An Approach to Automate and Optimize Concept Generation of Sheet Metal Parts by Topological and Parametric Decoupling' ASME. J. Mech. Des. May 2010; 132(5): 051001

[13] Gunwant, D., Misra, A. (2012) 'Topology Optimization of Sheet Metal Brackets Using ANSYS', MIT International Journal of Mechanical Engineering, 2(2), pp. 120-126.

[14] Hafiz, A. A. M., Hussein, H. M. A., Gemeal A. M. B., Naranje, V. (2019) 'Topology Optimization of Sheet Metal Combination Die', 2019 International Conference on Computational Intelligence and Knowledge Economy (ICCIKE), Dubai, United Arab Emirates, pp. 191-196

[15]Gupta, R. K., Sreenu, P., Bernard, A., Laroche, F. (2014) 'Process Information Model for Sheet Metal Operations', The IFIP Working Group WG 5.1 11th International Conference on Product Lifecycle Management, Jul 2014, Yokohama, Japan. ffhal-01312793f

[16] Opgenoord M. M. J., Willcox, K. E (2019) 'Design for additive manufacturing: cellular structures in early-stage aerospace design', Structural and Multidisciplinary Optimization, 60 (2) pp. 411 428. doi: 10.1007/s00158-019-02305-8

[17]Bendsøe, M. P. (1989) 'Optimal shape design as a material distribution problem' Structural Optimization, 1(4), pp. 193-202. , doi: 10.1007/BF01650949

[18]Fiebig, S., Sellschopp, J., Manz, H., Vietor, T., Axmann, K., Schumacher, A. (2015) 'Future challenges for topology optimization for the usage in automotive lightweight design technologies', Proc. of 11th World Congress on Structural and Multidisciplinary Optimization, Sydney, Australia 
[19] Additive Manufacturing and Production of Metallic Parts in Automotive Industry,” p. 123.

[20]Dijk, NP van, et al. (2013) 'Level-set methods for structural topology optimization: a review' Structural and Multidisciplinary Optimization. 48 (3), pp. 437-472.

[21]Hofmeyr, H.,. Davila Delgado, J.M. (2013) 'Automated Design Studies: Topology Versus OneStep Evolutionary Structural Optimization', Advanced Engineering Informatics, 27 (4), pp. 427 443.

[22]Huang, X., Xie, Y.M. (2008) 'Topology Optimization f Nonlinear Structures Under Displacement Loading', Engineering Structures, 30(7) pp. 2057-2068.

[23] Kalpak jian and Schmid (2008) Sheet-Metal Forming Processes. 1st ed. Pearson Education.

[24]Kubli, W., Heggen, A., Reissner, J. (1991) 'Nonlinear solver with uncoupled bending and stretching deformation for simulating thin sheet metal forming', VDI-Berichte $894-F E$ Simulation of 3-D Sheet Metal Forming Processes in Automotive Industry, VDI Verlag, Düsseldorf, pp. 325-343 